\title{
REGULARITY OF THE INVERSE OF A PLANAR SOBOLEV HOMEOMORPHISM
}

\author{
STANISLAV HENCL AND PEKKA KOSKELA
}

\section{INTRODUCTION}

In this paper we address the following issue. Suppose that $\Omega \subset \mathbb{R}^{2}$ is a domain and that $f: \Omega \rightarrow f(\Omega) \subset \mathbb{R}^{2}$ is a homeomorphism of the Sobolev class $W_{\text {loc }}^{1, p}\left(\Omega ; \mathbb{R}^{2}\right), p \geq 1$. Here $W_{\text {loc }}^{1, p}\left(\Omega ; \mathbb{R}^{2}\right)$ consists of all locally $p$-integrable mappings of $\Omega$ into $\mathbb{R}^{2}$ whose coordinate functions have locally $p$-integrable distributional derivatives. Under which conditions can we then conclude that $f^{-1} \in W_{\text {loc }}^{1,1}\left(f(\Omega), \mathbb{R}^{2}\right)$, or even that $f^{-1} \in W_{\text {loc }}^{1, q}\left(f(\Omega), \mathbb{R}^{2}\right)$ for some $q>1$ ?

Let us first briefly discuss the one-dimensional case. Consider the usual Cantor ternary function $u$ on the interval $(0,1)$. Then $u$ is continuous, non-decreasing, constant on each complementary interval of the ternary Cantor set and fails to be absolutely continuous. Let now $g(x)=x+u(x)$ on $(0,1)$. Then also $g$ fails to be absolutely continuous and so $g$ does not belong to $W_{\text {loc }}^{1,1}((0,1), \mathbb{R})$. On the other hand, the Lipschitz function $h=g^{-1}$ maps $(0,2)$ homeomorphically onto $(0,1)$. Thus, even the inverse of a Lipschitz homeomorphism $h$ can fail to belong to $W_{\text {loc }}^{1,1}$. If one analyzes the situation more carefully, one notices that the crucial thing here is that the differential of $h$ vanishes in a set of positive measure.

In dimension two, the mapping $f\left(x_{1}, x_{2}\right)=\left(h\left(x_{1}\right), x_{2}\right)$, where $h$ is as above, provides us with a Lipschitz homeomorphism whose inverse fails to belong to $W_{\text {loc }}^{1,1}$. Notice that the Jacobian determinant $J_{f}(x)$ of $f$ vanishes in a set of positive area. Our first result shows that this is the only situation where the inverse fails to belong to $W_{\text {loc }}^{1,1}$.

Theorem 1.1. Let $\Omega \subset \mathbb{R}^{2}$ be a domain. Suppose that $f \in W_{\mathrm{loc}}^{1,1}\left(\Omega, \mathbb{R}^{2}\right)$ is a homeomorphism and that $J_{f}(x)>0$ for a.e. $x \in \Omega$. Then $f^{-1} \in$ $W_{\text {loc }}^{1,1}\left(f(\Omega), \mathbb{R}^{2}\right)$ and

$$
\int_{f(\Omega)}\left|D f^{-1}(y)\right| d y \leq \int_{\Omega}|D f(x)| d x .
$$

The above theorem appears to substantially improve on the known related results. Indeed, the only results we know of assume that $f \in$

2000 Mathematics Subject Classification. 26B10, 30C65, 28A5, 46E35.

Key words and phrases. Sobolev mapping, Jacobian, inverse.

Both authors were supported in part by the Academy of Finland. 
$W_{\text {loc }}^{1,2}([30],[26])$. This is an essentially stronger assumption: it and the a.e. positivity of the Jacobian imply that $f$ maps sets of area zero to sets of area zero and even that the so-called distributional Jacobian of $f$ coincides with $J_{f}$. Both of these conclusions can fail in our setting, and our proof is necessarily different from the case $f \in W_{\text {loc }}^{1,2}$.

The statement of Theorem 1.1 is not symmetric in the sense that no conclusion on the size of the zero set of the Jacobian of $f^{-1}$ is drawn. In fact, under the setting of Theorem $1.1, J_{f^{-1}}$ can vanish in a set of positive area. Our second result extends Theorem 1.1 to an appealing statement.

Theorem 1.2. Let $\Omega \subset \mathbb{R}^{2}$ be a domain. Suppose that $f \in W_{\mathrm{loc}}^{1,1}\left(\Omega, \mathbb{R}^{2}\right)$ is a homeomorphism, and assume further that $D f(x)$ vanishes almost everywhere in the zero set of $J_{f}$. Then $f^{-1} \in W_{\mathrm{loc}}^{1,1}\left(f(\Omega), \mathbb{R}^{2}\right)$ and $D f^{-1}(y)$ vanishes almost everywhere in the zero set of $J_{f^{-1}}$. Moreover,

$$
\int_{f(\Omega)}\left|D f^{-1}(y)\right| d y=\int_{\Omega}|D f(x)| d x .
$$

Recall that a homeomorphism $f \in W_{\mathrm{loc}}^{1,1}(\Omega, f(\Omega))$ is classically differentiable almost everywhere. Thus either $J_{f}(x) \geq 0$ or $J_{f}(x) \leq 0$ a.e. For simplicity, let us assume from now on that $J_{f}(x) \geq 0$ a.e. Under the assumptions of Theorem 1.2, the inequality

$$
|D f(x)|^{2} \leq K(x) J_{f}(x)
$$

then holds a.e., where $1 \leq K(x)<\infty$ a.e. For simplicity, we then say that $f$ is a mapping (or homeomorphism) of finite distortion $K$ (cf. $[14]$ ). It is now natural to inquire if a suitable integrability condition on $K$ would guarantee better regularity for the inverse of $f$. Our next result gives an affirmative answer.

Theorem 1.3. Let $\Omega \subset \mathbb{R}^{2}$ be a domain. Suppose that $f \in W_{\mathrm{loc}}^{1,1}\left(\Omega, \mathbb{R}^{2}\right)$ is a homeomorphism of finite distortion such that $K \in L^{1}(\Omega)$. Then $f^{-1} \in W_{\mathrm{loc}}^{1,2}\left(f(\Omega), \mathbb{R}^{2}\right)$ and $f^{-1}$ is a mapping of finite distortion.

The first applications of the above results have already been found. In [13], we show that the $W^{1,1}$-minimizers of the $L^{1}$-integral of a suitable distortion function are in fact smooth, with a harmonic inverse. This relaxes the assumptions in [1], where the minimization problem was considered in $W^{1,2}$.

Based on Theorem 1.1 and Theorem 1.3, it would be natural to expect for an interpolation-type result, where the integrability of a power $0<a<1$ of $K$ would result in the $q$-integrability for $\left|D f^{-1}\right|$ for some $1<q(a)<2$. Surprisingly, this turns out not to be the case. Here and in the sequel, we use the notation $Q_{0}=[0,1] \times[0,1]$ for the unit square in $\mathbb{R}^{2}$. 
Example 1.4. Let $0<\delta<1$. There is a homeomorphism $f: Q_{0} \rightarrow Q_{0}$ of finite distortion such that $f \in W^{1,1}\left(Q_{0}, \mathbb{R}^{2}\right)$ and $K^{1-\delta} \in L^{1}\left(Q_{0}\right)$, but $f^{-1} \notin W_{\mathrm{loc}}^{1,1+\delta}\left(Q_{0}, \mathbb{R}^{2}\right)$.

Let us now compare our above results with previously known facts. First of all, it was known that $K \in L^{1}$ and $f \in W_{\text {loc }}^{1,2}\left(\Omega, \mathbb{R}^{2}\right)$ guarantee that $f^{-1} \in W_{\text {loc }}^{1,2}$, see [12]. In [22], it was further shown that the regularity assumption $f \in W_{\text {loc }}^{1,2}\left(\Omega, \mathbb{R}^{2}\right)$ can be slightly relaxed, say, to $|D f|^{2} \log ^{-1}(e+|D f|) \in L_{\text {loc }}^{1}$. These results were based on a duality argument, relying on integration by parts against the Jacobian determinant $J_{f}$, that does not work when one only assumes that $f \in W_{\text {loc }}^{1, p}\left(\Omega, \mathbb{R}^{2}\right)$ for some $p<2$. The approach used in [2], [30], [26], [8] to handle a problem arising from nonlinear elasticity is essentially the same. One could also try to apply the Beltrami equation. Such an approach was used in [16], but the necessary factorization property of the solutions basically requires that $f \in W_{\text {loc }}^{1,2}\left(\Omega, \mathbb{R}^{2}\right)$. There are also several recent papers where the existence of a homeomorphic solution $f$ with $f^{-1} \in W_{\text {loc }}^{1,2}$ to a Beltrami equation has been established under integrability conditions on the Beltrami coefficient [4], [3], [29], [11], [15]. The existence of such a solution does not necessarily guarantee that all homeomorphic solutions were as good, unless one has the factorization property.

The following corollary to our results shows that the regularity of the inverse of a solution to a Beltrami equation holds already under minimal assumptions.

Corollary 1.5. Let $\Omega \subset \mathbb{R}^{2}$ be a domain and let $f \in W_{\text {loc }}^{1,1}\left(\Omega, \mathbb{R}^{2}\right)$ be a homeomorphism with

$$
\partial f(z)=\mu(z) \bar{\partial} f(z)
$$

a.e. in $\Omega$, where $\mu$ is a complex valued function so that $|\mu(z)| \leq 1$ a.e. and $(1-|\mu(z)|)^{-1} \in L^{1}(\Omega)$. Then $f^{-1} \in W_{\text {loc }}^{1,2}\left(f(\Omega), \mathbb{R}^{2}\right)$ and

$$
\partial f^{-1}(z)=\tilde{\mu}(z) \bar{\partial} f^{-1}(z)
$$

a.e. in $f(\Omega)$, where $|\tilde{\mu}(z)|<1$ a.e.

Recall that the statement of Theorem 1.2 is symmetric. Thus one could inquire if some power of the distortion of $f^{-1}$ in Theorem 1.3 were also integrable. Our next result shows that such a conclusion holds (only) under exponential integrability of $K$.

Theorem 1.6. Let $\Omega \subset \mathbb{R}^{2}$ be a domain and let $f \in W_{\text {loc }}^{1,1}\left(\Omega, \mathbb{R}^{2}\right)$ be a homeomorphism of finite distortion. Assume that the distortion $K(x) \geq 1$ satisfies $\exp (\beta K) \in L_{\mathrm{loc}}^{1}(\Omega)$, for some $\beta>0$. Then $K_{f^{-1}}^{p} \in$ $L_{\mathrm{loc}}^{1}(f(\Omega))$, where $p=c_{2} \beta$ and $c_{2}>0$ is an absolute constant. Moreover, the claim fails for each $\beta$ for the value $c_{2}=2$.

Let us return to the lack of interpolation between Theorem 1.2 and Theorem 1.3. As Example 1.4 showed, the integrability of $K^{1-\delta}$ does 
not result in any better than $W^{1,1}$-regularity of $f^{-1}$, even when $\delta$ is small. One could still hope for some improvement under some a priori assumption on $f$. This turns out to be the case. We prove in Section 4 below that, given a homeomorphism $f \in W_{\text {loc }}^{1, p}\left(\Omega, \mathbb{R}^{2}\right), p>1$, of finite distortion with $K^{a} \in L^{1}(\Omega)$, one always has $f^{-1} \in W_{\text {loc }}^{1, q}\left(f(\Omega), \mathbb{R}^{2}\right)$, where $1<q(p, a) \leq 2$. The obtained exponent $q$ turns out to be sharp. This is proven in Section 7 by means of a novel construction. In the special case when $f$ is Lipschitz, one can take $q=a+1$, for $0<a \leq 1$. There is no additional gain from an exponent $a>1$ : simply notice that the Lipschitz homeomorphism

$$
f(x)=x\|x\|^{s}
$$

has a bounded $K$ for each (large) $s>0$ and $f^{-1} \notin W_{\text {loc }}^{1, q}$ for $q=2+2 / s$.

We close this introduction by pointing out an application of Theorem 1.3 that will be discussed in more detail in Section 6. It follows from Theorem 1.3 that $f^{-1} \in W_{\text {loc }}^{1,2}\left(f(\Omega), \mathbb{R}^{2}\right)$ with $J_{f^{-1}} \geq 0$ a.e. whenever $f \in W_{\text {loc }}^{1,1}\left(\Omega, \mathbb{R}^{2}\right)$ is a homeomorphism of finite distortion with $K \in$ $L^{1}(\Omega)$. By a result from [25], this guarantees that $J_{f^{-1}} \log \left(e+J_{f^{-1}}\right)$ is locally integrable. It follows that $\log \left(e+\frac{1}{J_{f}}\right) \in L_{\mathrm{loc}}^{1}(\Omega)$, and this conclusion turns out to be essentially sharp. Previously it was only known (cf. [21]) that these assumptions guarantee that $J_{f}(x)>0$ a.e. Our methods are flexible enough to produce further extensions of this phenomenon.

\section{Preliminaries}

2.1. Notation. For $c=\left(c_{1}, c_{2}\right) \in \mathbb{R}^{2}, a>0$ and $b>0$ we write $R(c, a, b)=\left[c_{1}-a, c_{1}+a\right] \times\left[c_{2}-b, c_{2}+b\right]$; then $R(c, a, b)$ is a rectangle in $\mathbb{R}^{2}$. By $B(x, r)$ we denote the two-dimensional open ball centered at $x \in \mathbb{R}^{2}$ with radius $r>0$. In the entire paper, $\Omega$ will denote a domain in $\mathbb{R}^{2}$.

We use the notation $\mathbf{e}_{1}=(1,0)$ and $\mathbf{e}_{2}=(0,1)$ for the unit vectors. For $a \in \mathbb{R}^{2}$ we write $(a)_{1}$ and $(a)_{2}$ for its coordinates, i.e. $a=(a)_{1} \mathbf{e}_{1}+$ $(a)_{2} \mathbf{e}_{2}$.

We write $\|x\|$ for the euclidean norm of $x \in \mathbb{R}^{2}$. Given a square matrix $B$ we define the norm $|B|$ as the supremum of $\|B x\|$ over all vectors $x$ of unit euclidean norm. Recall that the adjugate adj $B$ of $B$ satisfies

$$
B \operatorname{adj} B=I \operatorname{det}(B),
$$

where $\operatorname{det}(B)$ denotes the determinant of $B$ and $I$ is the identity matrix. We denote the Lebesque measure of a measurable set $E \subset \mathbb{R}^{2}$ by $|E|$. Given two functions $F, G: \Omega \rightarrow \mathbb{R}$ we write $G \sim F$ if there is a constant $C \geq 1$ such that $F(x) / C \leq G(x) \leq C F(x)$ for every $x \in \Omega$. 
By int $A$ we denote the interior of a set $A \subset \mathbb{R}^{2}$. We use the notation $\operatorname{sgn}$ for the sign function, i.e. $\operatorname{sgn}(t)=1$ if $t>0$ and $\operatorname{sgn}(t)=-1$ if $t<0$.

2.2. Differentiability of $W^{1,1}$-mappings. Every continuous mapping $f \in W^{1,1}\left(\Omega, \mathbb{R}^{2}\right)$ has finite partial derivatives almost everywhere (because it is absolutely continuous on almost every line parallel to coordinate axes, see e.g. [28, I.1.2]), and therefore we have the following corollary to $[9$, Theorem 2].

Theorem 2.1. Let $f \in W_{\text {loc }}^{1,1}\left(\Omega, \mathbb{R}^{2}\right)$ be a homeomorphism. Then $f$ is differentiable almost everywhere in $\Omega$.

2.3. Area formula. Let $A \subset \Omega$ be a measurable set. Assume that $g$ is a homeomorphism such that $g$ is differentiable at every point of $A$. Let $\eta$ be a non-negative Borel-measurable function on $\mathbb{R}^{2}$. Then

$$
\int_{A} \eta(g(x))\left|J_{g}(x)\right| d x \leq \int_{\mathbb{R}^{2}} \eta(y) d y .
$$

This follows from [7, Theorem 3.1.8] together with the area formula for Lipschitz mappings. In particular, given a homeomorphism $g \in$ $W_{\text {loc }}^{1,1}\left(\Omega ; \mathbb{R}^{2}\right)$, we obtain with the help of Theorem 2.1 that

$$
\int_{\Omega} \eta(g(x))\left|J_{g}(x)\right| d x \leq \int_{\mathbb{R}^{2}} \eta(y) d y .
$$

2.4. Properties of integrable functions. The following sufficient condition for $L^{1}$-weak compactness will be needed (for the proof see e.g. [5] or [23, Lemma 7.11]).

Lemma 2.2. Let $\left(g_{j}\right)_{j \in \mathbb{N}}$ be a sequence of measurable functions on a domain $\Omega \subset \mathbb{R}^{2}$ of finite measure. Suppose that there is $H \in L^{1}(\Omega)$ such that for almost every $y \in \Omega$ and for every $j \in \mathbb{N}$ we have $\left|g_{j}(y)\right| \leq$ $H(y)$. Then there is a subsequence $\left(\tilde{g}_{j}\right)_{j \in \mathbb{N}}$ of $\left(g_{j}\right)_{j \in \mathbb{N}}$ and $g \in L^{1}(\Omega)$ such that the subsequence $\left(\tilde{g}_{j}\right)_{j \in \mathbb{N}}$ converges weakly to $g$ in $L^{1}(\Omega)$.

Let $h \in L^{1}(\Omega)$. It is a well-known fact that the Lebesgue integral is absolutely continuous with respect to the Lebesgue measure, i.e. for every $\varepsilon>0$ there is $\delta>0$ such that

$$
\text { for every } E \subset \Omega \text { we have }\left[|E|<\delta \Rightarrow \int_{E} h<\varepsilon\right] \text {. }
$$

2.5. Derivative of a radial stretching. The following lemma can be verified by an elementary calculation.

Lemma 2.3. Let $\rho:(0, \infty) \rightarrow(0, \infty)$ be a strictly monotone, differentiable function. Then for the mapping

$$
f(x)=\frac{x}{\|x\|} \rho(\|x\|), \quad x \neq 0
$$


we have for almost every $x$

$$
D f(x) \sim \max \left\{\frac{\rho(\|x\|)}{\|x\|},\left|\rho^{\prime}(\|x\|)\right|\right\}, J_{f}(x) \sim \frac{\rho^{\prime}(\|x\|) \rho(\|x\|)}{\|x\|} .
$$

\section{Regularity of THE INVERSE MAPPING}

Lemma 3.1. Suppose that $f \in W_{\mathrm{loc}}^{1,1}\left(\Omega, \mathbb{R}^{2}\right)$ is a homeomorphism and let $B(y, 2 r) \subset \subset f(\Omega)$. Then

$$
r \operatorname{diam} f^{-1}(B(y, r)) \leq \int_{f^{-1}(B(y, 2 r))}|D f| .
$$

Proof. Set $d=\operatorname{diam} f^{-1}(B(y, r))$ and pick $a, b \in f^{-1}(B(y, r))$ such that $|a-b|=d$. Without loss of generality, we will suppose that $a=(0,0)$ and $b=(d, 0)$. For $t \in[0, d]$ we denote

$$
L_{t}=\left\{s \in \mathbb{R}:(t, s) \in f^{-1}(B(y, 2 r))\right\} .
$$

Since $f$ is absolutely continuous on almost every line parallel to the $y$-axis and $\operatorname{diam} f\left(\{t\} \times L_{t}\right) \geq r$ we obtain

$$
\int_{L_{t}}|D f(t, s)| d s \geq r
$$

for almost every $t \in[0, d]$. By integrating this inequality over $[0, d]$ we obtain (3.1).

In the next theorem, we will show that $f^{-1} \in W_{\text {loc }}^{1,1}$. We will first construct approximations to $f^{-1}$ and then, using Lemma 2.2, we will prove that these approximations converge weakly to $f^{-1}$.

Theorem 3.2. Suppose that $f \in W_{\text {loc }}^{1,1}\left(\Omega, \mathbb{R}^{2}\right)$ is a homeomorphism of finite distortion. Then $f^{-1} \in W_{\mathrm{loc}}^{1,1}\left(f(\Omega), \mathbb{R}^{2}\right)$.

Proof. Let $A \subset \subset f(\Omega)$ be a fixed domain. First we will construct approximations to $f^{-1}$. Fix $\varepsilon>0$ such that $\{x: \operatorname{dist}(x, A)<10 \varepsilon\} \subset \subset$ $\Omega$.

We denote the $\varepsilon$-grid in $\mathbb{R}^{2}$ by $G_{\varepsilon}=(\varepsilon \mathbb{Z}) \times(\varepsilon \mathbb{Z})$. Pick a partition of unity $\left\{\phi_{z}\right\}_{z \in G_{\varepsilon}}$ such that

$$
\text { each } \phi_{z}: \mathbb{R}^{2} \rightarrow \mathbb{R} \text { is continuously differentiable; }
$$

$$
\begin{aligned}
& \operatorname{supp} \phi_{z} \subset B(z, 2 \varepsilon) \text { and }\left|\nabla \phi_{z}\right| \leq \frac{C}{\varepsilon} \\
& \sum_{z \in G_{\varepsilon}} \phi_{z}(y)=1 \text { for every } y \in \mathbb{R}^{2} .
\end{aligned}
$$

Now we set

$$
g_{\varepsilon}(y)=\sum_{z \in G_{\varepsilon}} \phi_{z}(y) f^{-1}(z) \text { for every } y \in A
$$


This approximation to $f^{-1}$ clearly satisfies $g_{\varepsilon} \in C^{1}\left(A, \mathbb{R}^{2}\right)$. Then

$$
\left|D g_{\varepsilon}(y)\right| \leq \frac{C}{\varepsilon} \operatorname{diam} f^{-1}(B(y, 2 \varepsilon)) \text {. }
$$

Indeed, for fixed $y$, choose $z_{0}$ so that $y \in B\left(z_{0}, 2 \varepsilon\right)$. Then

$$
D g_{\varepsilon}(y)=D \sum_{z \in G_{\varepsilon}} \phi_{z}(y)\left(f^{-1}(z)-f^{-1}\left(z_{0}\right)\right)
$$

because of (3.2), and the asserted estimate follows. Together with Lemma 3.1 this implies that for every $y \in A$ we have

$$
\left|D g_{\varepsilon}(y)\right| \leq \frac{C}{\varepsilon^{2}} \int_{f^{-1}(B(y, 4 \varepsilon))}|D f(x)| d x .
$$

Denote $\tilde{G}=\left\{x \in \Omega: f\right.$ is differentiable at $x$ and $\left.J_{f}(x)>0\right\}$. Since $f$ is mapping of finite distortion (i.e. $J_{f}(x)=0 \Rightarrow D f(x)=0$ a.e.) we conclude with the help of Theorem 2.1 that $D f(x)=0$ a.e. in $\Omega \backslash \tilde{G}$. Pick a Borel set $G \subset \tilde{G}$ such that $|G|=|\tilde{G}|$. From (3.3) and (2.2) we now have

$$
\begin{aligned}
\left|D g_{\varepsilon}(y)\right| & \leq \frac{C}{\varepsilon^{2}} \int_{f^{-1}(B(y, 4 \varepsilon)) \cap G}|D f(x)| d x \\
& \leq \frac{C}{\varepsilon^{2}} \int_{B(y, 4 \varepsilon) \cap f(G)} \frac{\left|(D f)\left(f^{-1}(z)\right)\right|}{J_{f}\left(f^{-1}(z)\right)} d z .
\end{aligned}
$$

We claim that

$$
F(z):=\frac{\left|(D f)\left(f^{-1}(z)\right)\right|}{J_{f}\left(f^{-1}(z)\right)} \chi_{f(G)}(z) \in L^{1}(A) .
$$

Note that $f(G)$ is a Borel set (as a preimage of a Borel set under the continuous map $f^{-1}$ ) and hence $F$ is measurable. For every $z \in f(G)$ we know that $f$ is differentiable at $f^{-1}(z)$ and that $J_{f}\left(f^{-1}(z)\right)>0$. Therefore $f^{-1}$ is differentiable at $z$ and $J_{f^{-1}}(z)=1 / J_{f}\left(f^{-1}(z)\right)$. It follows from (2.1) for $g=f^{-1}$ that

$$
\int_{A} F(z) d z=\int_{A \cap f(G)}\left|(D f)\left(f^{-1}(z)\right)\right| J_{f^{-1}}(z) d z \leq \int_{f^{-1}(A) \cap G}|D f|<\infty .
$$

Since $f_{B(y, 4 \varepsilon)} F \rightarrow F(y)$ in $L^{1}(A)$ as $\varepsilon \rightarrow 0$, there is a subsequence $\varepsilon_{j} \rightarrow 0$ such that $f_{B\left(y, 4 \varepsilon_{j}\right)} F$ has a majorant $H \in L^{1}(A)$. From this, (3.4) and Lemma 2.2, we obtain that there is a subsequence $\varepsilon_{i} \rightarrow 0$ and $g \in L^{1}\left(A, \mathbb{R}^{2}\right)$ such that $D g_{\varepsilon_{i}} \rightarrow g$ weakly in $L^{1}(A)$. Clearly

$$
\int_{A} D g_{\varepsilon_{i}}(y) \phi(y) d y=-\int_{A} g_{\varepsilon_{i}}(y) D \phi(y) d y
$$

for every test function $\phi \in C_{c}^{\infty}\left(A, \mathbb{R}^{2}\right)$. Since $g_{\varepsilon} \rightarrow f^{-1}$ locally uniformly as $\varepsilon \rightarrow 0$, we obtain, after passing to a limit, that

$$
\int_{A} g(y) \phi(y) d y=-\int_{A} f^{-1}(y) D \phi(y) d y
$$


which means that $g$ is a weak gradient of $f^{-1}$ in $A$ and therefore $f^{-1} \in$ $W^{1,1}(A)$.

Theorem 3.3. Suppose that $f \in W_{\mathrm{loc}}^{1,1}\left(\Omega, \mathbb{R}^{2}\right)$ is a homeomorphism of finite distortion. Then $f^{-1}$ is a mapping of finite distortion.

Proof. From Theorem 3.2 we know that $f^{-1} \in W_{\text {loc }}^{1,1}\left(f(\Omega), \mathbb{R}^{2}\right)$. Suppose that $f^{-1}$ is not a mapping of finite distortion. Then we can find a measurable set $M$ and an open set $A$ such that $M \subset A \subset \subset f(\Omega)$, $|A|<\infty,|M|>0$, and

$$
\text { for every } x \in M \text { we have }\left|D f^{-1}(x)\right|>0 \text { and } J_{f^{-1}}(x)=0 \text {. }
$$

Thus there exists $k \in \mathbb{Z}$ such that for

$$
\tilde{M}=\left\{x \in M: 2^{k}<\left|D f^{-1}(x)\right| \leq 2^{k+1}\right\} \text { we have }|\tilde{M}|>0 .
$$

In view of Theorem 2.1, we may moreover assume that $f^{-1}$ is differentiable at every point of $\tilde{M}$. Let $\eta>0$. From (3.6) and (3.7) we obtain that for every $x \in \tilde{M}$ we can pick a ball $B(x, r(x))$ such that

$$
\begin{aligned}
& B(x, 2 r(x)) \subset A, r(x)<1, \\
& \operatorname{diam} f^{-1}(B(x, r(x)))>2^{k} r(x) \text { and } \\
& \left|f^{-1}(B(x, 2 r(x)))\right|<\eta|B(x, 2 r(x))| .
\end{aligned}
$$

We use the Vitali covering theorem for the family $\{B(x, 2 r(x))\}_{x \in \tilde{M}}$ to obtain balls $B_{i}:=B\left(x_{i}, r_{i}\right)$ such that

$$
\tilde{M} \subset \bigcup_{i} 10 B_{i} \text { and } 2 B_{i} \text { are pairwise disjoint. }
$$

Hence (3.9), Lemma 3.1 and (3.8) give us

$$
\begin{aligned}
|\tilde{M}| & \leq 10^{n} \sum_{i}\left|B_{i}\right| \leq C(n) \sum_{i} r_{i}^{2} \leq \\
& \leq C(n) 2^{-k} \sum_{i} r_{i} \operatorname{diam}\left(f^{-1}\left(B\left(x_{i}, r_{i}\right)\right)\right) \leq \\
& \leq C(n, k) \sum_{i} \int_{f^{-1}\left(2 B_{i}\right)}|D f|=C(n, k) \int_{\bigcup_{i} f^{-1}\left(2 B_{i}\right)}|D f| .
\end{aligned}
$$

By (3.8) we conclude that

$$
\left|\bigcup_{i} f^{-1}\left(2 B_{i}\right)\right| \leq \eta \sum_{i}\left|2 B_{i}\right| \leq \eta|A| \stackrel{\eta \rightarrow 0+}{\rightarrow} 0 .
$$

This fact, (3.10) and the assumption $D f \in L_{\text {loc }}^{1}(\Omega)$ contradict (2.3).

Proof of Theorem 1.2. From Section 4, Chapter I in [28] we know that each homeomorphism $f: \Omega \rightarrow \mathbb{R}^{2}$ is either sense-preserving or sensereversing. Without loss of generality, we will suppose that $f$ is sensepreserving. Thus, at every point of differentiability of $f$, we have that $J_{f}(x) \geq 0$ (see [28, Lemma 4.11]). Together with Lemma 2.1 this implies that $J_{f}(x) \geq 0$ a.e. and thus $f$ is a mapping of finite distortion. 
REGULARITY OF THE INVERSE OF A PLANAR HOMEOMORPHISM 9

Relying on Theorem 3.2 and Theorem 3.3, we thus conclude that $f^{-1} \in$ $W_{\text {loc }}^{1,1}(f(\Omega), \Omega)$ has finite distortion. Therefore we may use Theorem 2.1 to conclude that

$$
\int_{f(\Omega)}\left|D f^{-1}(y)\right| d y=\int_{A}\left|D f^{-1}(y)\right| d y
$$

where $A$ is a Borel subset of the set $E$ where $f^{-1}$ is differentiable with $J_{f^{-1}}>0$ such that $|A|=|E|$. Applying (2.1) and basic linear algebra we arrive at

$$
\begin{gathered}
\int_{A}\left|D f^{-1}(y)\right| d y=\int_{A} \frac{\left|D f^{-1}(y)\right|}{J_{f^{-1}}(y)} J_{f^{-1}}(y) d y \\
\leq \int_{f^{-1}(A)} \frac{\left|D f^{-1}(f(x))\right|}{J_{f^{-1}}(f(x))} d x=\int_{f^{-1}(A)}\left|(D f(x))^{-1}\right| J_{f}(x) d x \\
=\int_{f^{-1}(A)}|\operatorname{adj} D f(x)| d x \leq \int_{\Omega}|D f(x)| d x .
\end{gathered}
$$

We conclude that

$$
\int_{f(\Omega)}\left|D f^{-1}\right| \leq \int_{\Omega}|D f|
$$

The opposite inequality follows by symmetry; recall that also $f$ has finite distortion.

Proof of Theorem 1.1. Theorem 1.1 is an easy consequence of Theorem 1.2.

\section{HigheR REgUlarity OF THE INVERSE MAPPING}

Proof of Theorem 1.3. Analogously to (3.11) and (3.12) we deduce that

$$
\begin{gathered}
\int_{f(\Omega)}\left|D f^{-1}(y)\right|^{2} d y \leq \int_{f^{-1}(A)} \frac{\left|D f^{-1}(f(x))\right|^{2}}{J_{f^{-1}}(f(x))} d x= \\
=\int_{f^{-1}(A)}\left|(D f(x))^{-1}\right|^{2} J_{f}(x) d x=\int_{f^{-1}(A)} \frac{|\operatorname{adj} D f(x)|^{2}}{J_{f}(x)} d x \leq \int_{\Omega} K .
\end{gathered}
$$

We are now in position to prove an interpolation-type result.

Theorem 4.1. Let $p \in(1, \infty], 1<q<2$ and set $a=\frac{(q-1) p}{p+q-2}$ (for $r=\infty$ we set $a=(q-1))$. Let $\Omega \subset \mathbb{R}^{2}$ be a domain and suppose that $f \in W_{\mathrm{loc}}^{1, p}\left(\Omega, \mathbb{R}^{2}\right)$ is a homeomorphism of finite distortion such that $K^{a} \in L^{1}(\Omega)$. Then $f^{-1} \in W_{\text {loc }}^{1, q}\left(f(\Omega), \mathbb{R}^{2}\right)$.

Proof. We again reason as for (3.11) and (3.12) and use Hölder's inequality to conclude that

$$
\int_{f(\Omega)}\left|D f^{-1}(y)\right|^{q} d y \leq \int_{f^{-1}(A)} \frac{|\operatorname{adj} D f(x)|^{q}}{J_{f}(x)^{q-1}} d x \leq
$$




$$
\leq \int_{f^{-1}(A)}|D f(x)|^{2-q} K(x)^{q-1} d x \leq\|D f\|_{L^{p}(\Omega)}^{2-q}\left(\int_{\Omega} K^{a}\right)^{\frac{p+q-2}{p}} .
$$

In certain questions the $L^{2}$-integrability assumption of partial derivatives can be relaxed to a slightly weaker assumption. Such a situation will occur in Theorem 6.1 (ii) below; also see [20]. The following gives a sufficient condition for an integrability condition of this type for $f^{-1}$.

Theorem 4.2. Let $\Phi:[0, \infty) \rightarrow[1, \infty)$ be an increasing function such that for every $A>0$ there is $C(A)>0$ such that

$$
\Phi\left(t^{A}\right)>C(A) \Phi(t) \text { for every } t>0 .
$$

Suppose that $f \in W_{\mathrm{loc}}^{1,1}\left(\Omega, \mathbb{R}^{2}\right)$ is a homeomorphism of finite distortion. Suppose that $\frac{K}{\Phi(K)} \in L^{1}(\Omega)$ and $|D f|^{p} \in L^{1}(\Omega)$ for some $p>1$. Then $f^{-1} \in W_{\mathrm{loc}}^{1,1}\left(f(\Omega), \mathbb{R}^{2}\right)$ and

$$
\frac{\left|D\left(f^{-1}\right)\right|^{2}}{\Phi\left(\left|D\left(f^{-1}\right)\right|\right)} \in L^{1}(f(\Omega)) .
$$

Proof. Analogously to (3.11) and (3.12) we obtain by (4.1) that

$$
\begin{aligned}
& \int_{f(\Omega)} \frac{\left|D f^{-1}(y)\right|^{2}}{\Phi\left(\left|D\left(f^{-1}\right)\right|\right)} d y \leq \int_{f^{-1}(A)} \frac{|\operatorname{adj} D f(x)|^{2}}{J_{f}(x) \Phi\left(\frac{|\operatorname{adj} D f(x)|}{J_{f}(x)}\right)} d x \leq \\
\leq & \int_{f^{-1}(A)} \frac{K(x)}{\Phi\left(\frac{K(x)}{|D f(x)|}\right)} d x \leq \int_{\left\{K(x) \leq|D|^{p}\right\}}+\int_{\left\{\frac{K(x)}{|D f(x)|}>K(x)^{1-\frac{1}{p}}\right\}} \leq \\
\leq & \int_{f^{-1}(A)}|D f(x)|^{p} d x+\frac{1}{C\left(1-\frac{1}{p}\right)} \int_{f^{-1}(A)} \frac{K(x)}{\Phi(K(x))} d x<\infty .
\end{aligned}
$$

\section{Integrability of $K_{f^{-1}}$}

Lemma 5.1. Let $a>0, b>0, \alpha>0$ and $\beta>0$. Then

$$
a^{\frac{\alpha}{2}} b \leq b \log ^{\alpha}(e+b)+C(\alpha, \beta) \exp (\beta a) .
$$

Proof. If the first term on the right-hand side of (5.1) is greater or equal to the left-hand side, then the inequality is obvious. Otherwise, $b \leq \exp (\sqrt{a})$, which implies that

$$
a^{\frac{\alpha}{2}} b \leq a^{\frac{\alpha}{2}} \exp (\sqrt{a}) \leq C(\alpha, \beta) \exp (\beta a) .
$$

We will employ the following result on the higher integrability of the Jacobian of a mapping of finite distortion from [6, Theorem 1.1] (see also [4]). 
Theorem 5.2. Let $f \in W_{\mathrm{loc}}^{1,1}\left(\Omega, \mathbb{R}^{2}\right)$ be a mapping of finite distortion. Assume that the distortion $K(x) \geq 1$ satisfies $\exp (\beta K) \in L_{\text {loc }}^{1}(\Omega)$, for some $\beta>0$. Then

$$
J_{f}(x) \log ^{\alpha}\left(e+J_{f}(x)\right) \in L_{\mathrm{loc}}^{1}(\Omega),
$$

where $\alpha=c_{1} \beta$ and $c_{1}$ is an absolute constant.

Proof of Theorem 1.6. From Theorem 3.2 and Theorem 3.3 we know that $f^{-1}$ is a mapping of finite distortion. Set $c_{2}=\frac{c_{1}}{2}$ where $c_{1}$ comes from Theorem 5.2 and let $A$ be any compact subset of $f(\Omega)$ such that for every $y \in A, f^{-1}$ is differentiable at $y$ with $J_{f^{-1}}(y)>0$. Analogously to the proof of Theorem 1.3 we obtain using (5.1) and choosing $p=\frac{c_{1}}{2} \beta$ that

$$
\begin{aligned}
\int_{A} K_{f^{-1}}^{p} & \leq \int_{f^{-1}(A)} \frac{\left|D\left(f^{-1}\right)(f(x))\right|^{2 p}}{J_{f^{-1}}(f(x))^{p+1}} d x \\
& \leq \int_{f^{-1}(A)} \frac{|\operatorname{adj} D f(x)|^{2 p}}{J_{f}(x)^{2 p} J_{f^{-1}}(f(x))^{p+1}} d x \\
& \leq \int_{f^{-1}(A)} K(x)^{p} J_{f}(x) d x \\
& \leq \int_{f^{-1}(A)}\left(J_{f}(x) \log ^{c_{1} \beta}\left(e+J_{f}(x)\right)+C \exp (\beta K(x))\right) d x .
\end{aligned}
$$

Since $f^{-1}$ is a mapping of finite distortion we can define $K_{f^{-1}}(y)=1$ for every $y \in f(\Omega)$ such that $J_{f^{-1}}(y)=0$. Then Theorem 2.1, Theorem 5.2 and (5.2) imply $K_{f^{-1}}^{p} \in L_{\text {loc }}^{1}(f(\Omega))$.

To see that we cannot take $c_{2}=2$, consider the mapping defined by setting $f(0)=0$ and

$$
f(x)=\frac{x}{\|x\|} \frac{1}{\log ^{a} \frac{1}{\|x\|}} \text { for } x \in B\left(0, \frac{1}{e}\right) \backslash\{0\} .
$$

Then $f \in W^{1,1}\left(B\left(0, \frac{1}{e}\right), \mathbb{R}^{2}\right)$ is a homeomorphism of finite distortion with $\exp \left(a K_{f}\right) \in L^{1}\left(B\left(0, \frac{1}{e}\right)\right)$, but $K_{f^{-1}}^{2 a} \notin L^{1}(B(0,1))$.

Indeed, from Lemma 2.3 we obtain that for $\|x\|<\exp (-a)$ we have

$$
\begin{gathered}
D f(x) \sim \max \left\{\frac{1}{\|x\| \log ^{a} \frac{1}{\|x\|}}, \frac{a}{\|x\| \log ^{a+1} \frac{1}{\|x\|}}\right\}=\frac{1}{\|x\| \log ^{a} \frac{1}{\|x\|}}, \\
J_{f}(x) \sim \frac{a}{\|x\|^{2} \log ^{2 a+1} \frac{1}{\|x\|}} \text { and hence } K_{f}(x) \sim \frac{1}{a} \log \frac{1}{\|x\|} .
\end{gathered}
$$

It follows that $f \in W^{1,1}\left(B\left(0, \frac{1}{e}\right), \mathbb{R}^{2}\right)$ and $\exp \left(a K_{f}\right) \in L^{1}\left(B\left(0, \frac{1}{e}\right)\right)$.

Then we can use Lemma 2.3 for $f^{-1}: B(0,1) \rightarrow B\left(0, \frac{1}{e}\right)$ which is clearly given by

$$
f^{-1}(y)=\frac{y}{\|y\|} \exp \left(\frac{-1}{\|y\|^{1 / a}}\right) \text { for } y \neq 0
$$


to obtain

$$
\begin{gathered}
\left|D f^{-1}(y)\right| \sim \max \left\{\frac{\exp \left(\frac{-1}{\|y\|^{1 / a}}\right)}{\|y\|}, \frac{\exp \left(\frac{-1}{\|y\|^{1 / a}}\right)}{a\|y\|^{\frac{1}{a}+1}}\right\} \sim C(a) \frac{\exp \left(\frac{-1}{\|y\|^{1 / a}}\right)}{\|y\|^{\frac{1}{a}+1}}, \\
J_{f^{-1}}(y) \sim C(a) \frac{\exp \left(\frac{-2}{\|y\|^{1 / a}}\right)}{\|y\|^{\frac{1}{a}+2}}, \text { and hence } K_{f^{-1}}(y) \sim \frac{C(a)}{\|y\|^{\frac{1}{a}}} .
\end{gathered}
$$

An elementary computation now gives us $K_{f^{-1}}^{2 a} \notin L^{1}(B(0,1))$.

Notice that the mapping $f \in W^{1,1}\left(B\left(0, \frac{1}{e^{2}}\right), \mathbb{R}^{2}\right)$ defined by $f(0)=0$ and

$$
f(x)=\frac{x}{\|x\|} \frac{1}{\log \log \frac{1}{\|x\|}} \text { for } x \neq 0
$$

in a small neigborhood of the origin satisfies $K_{f} \in L^{p}\left(B\left(0, \frac{1}{e^{2}}\right)\right)$ for every $p \in[1, \infty)$, but $K_{f^{-1}}^{q} \notin L^{1}(B(0,1))$ for any $q>0$. Thus no $p$-integrability of $K$ can guarantee any $q$-integrability of $K_{f^{-1}}$.

\section{INTEGRABILITY OF $\frac{1}{J_{f}}$}

Theorem 6.1. Let $\Omega \subset \mathbb{R}^{2}$ be a domain.

(i) Suppose that $f \in W_{\mathrm{loc}}^{1,1}\left(\Omega, \mathbb{R}^{2}\right)$ is a homeomorphism of finite distortion such that $K \in L^{1}(\Omega)$. Then $J_{f}>0$ a.e. in $\Omega$ and $\log \left(e+\frac{1}{J_{f}}\right) \in$ $L_{\text {loc }}^{1}(\Omega)$.

(ii) Let $p>1$ and suppose that $f \in W_{\text {loc }}^{1, p}\left(\Omega, \mathbb{R}^{2}\right)$ is a homeomorphism of finite distortion such that $K \log ^{-1}(e+K) \in L^{1}(\Omega)$. Then $J_{f}>0$ a.e. in $\Omega$ and $\log \log \left(e+\frac{1}{J_{f}}\right) \in L_{\mathrm{loc}}^{1}(\Omega)$.

Proof. ( $i$ ) From our assumptions and Theorem 1.3 we obtain $f^{-1} \in$ $W_{\text {loc }}^{1,2}\left(f(\Omega), \mathbb{R}^{2}\right)$, and thus $J_{f^{-1}} \log \left(e+J_{f^{-1}}\right) \in L_{\text {loc }}^{1}(f(\Omega))$ (see $\left.[25]\right)$. We denote by $\tilde{A} \subset \Omega$ the set where $f$ is differentiable with $J_{f}>0$. From [21] and Theorem 2.1 we know that $|\tilde{A}|=|\Omega|$ and therefore we may find a Borel set $A \subset \tilde{A}$ such that $|A|=|\Omega|$. Hence we may use (2.2) and $\frac{1}{J_{f}(x)}=J_{f^{-1}}(f(x))$ for $x \in A$ to obtain

$$
\begin{aligned}
\int_{E} \log \left(e+\frac{1}{J_{f}(x)}\right) d x & =\int_{E \cap A} \frac{1}{J_{f}(x)} \log \left(e+\frac{1}{J_{f}(x)}\right) J_{f}(x) d x \\
& \leq \int_{f(E \cap A)} J_{f^{-1}}(y) \log \left(e+J_{f^{-1}}(y)\right) d y<\infty
\end{aligned}
$$

for each compact set $E \subset \Omega$.

(ii) From Theorem 4.2 we obtain that

$$
\frac{\left|D f^{-1}\right|^{2}}{\log \left(e+\left|D f^{-1}\right|\right)} \in L_{\mathrm{loc}}^{1}(f(\Omega))
$$


REGULARITY OF THE INVERSE OF A PLANAR HOMEOMORPHISM 13

and therefore $J_{f^{-1}} \log \log \left(e+J_{f^{-1}}\right) \in L_{\text {loc }}^{1}(f(\Omega))$ (see [24]). Because $f^{-1}$ is a homeomorphism, we may thus use [18, Theorem A] to conclude that $f^{-1}$ satisfies condition $\mathrm{N}$, that is, $f^{-1}$ maps sets of measure zero to sets of measure zero.

We claim that $J_{f}>0$ a.e. in $\Omega$. If not, then we may use Theorem 2.1 to see that the set

$$
M=\left\{x \in \Omega: J_{f}(x)=0 \text { and } f \text { is differentiable at } x\right\}
$$

has positive measure. Then the differentiability and a simple covering argument shows that $|f(M)|=0$, which contradicts condition $\mathrm{N}$ for $f^{-1}$. Analogously to (6.1) we now conclude that

$$
\begin{aligned}
\int_{E} \log \log \left(e+\frac{1}{J_{f}(x)}\right) d x & =\int_{E \cap A} \frac{1}{J_{f}(x)} \log \log \left(e+\frac{1}{J_{f}(x)}\right) J_{f}(x) d x \\
& \leq \int_{f(E \cap A)} J_{f^{-1}}(y) \log \log \left(e+J_{f^{-1}}(y)\right) d y<\infty
\end{aligned}
$$

for each compact set $E \subset \Omega$.

Analogously to the proof of part (ii) above, it would be possible to use [14, Theorem 8.4.1] to obtain further results on the integrability of $\frac{1}{J_{f}}$ also under weaker conditions, like $K \log ^{-1}(e+K) \log ^{-1} \log (e+K) \in$ $L^{1}$ and so on. The following two examples from [17] show that the assumptions of Theorem 6.1 are sharp.

Example 6.2. Let $\varepsilon>0$. There exists a Lipschitz homeomorphism $f: Q_{0} \rightarrow Q_{0}$ of finite distortion such that $\frac{K}{\log ^{1+\varepsilon}(e+K)} \in L^{1}\left(Q_{0}\right)$ and $J_{f}=0$ on a set of positive measure.

Example 6.3. Let $\alpha:(0, \infty) \rightarrow[1, \infty)$ be a continuous, increasing function such that $\lim _{t \rightarrow \infty} \alpha(t)=\infty$. There exists a homeomorphism $f \in W^{1,1}\left(Q_{0}, \mathbb{R}^{2}\right)$ of finite distortion such that $\frac{K}{\alpha(K)} \in L^{1}\left(Q_{0}\right)$ and $J_{f}=0$ on a set of positive measure.

The last example of this section shows that the statement of Theorem $6.1(i)$ is sharp.

Example 6.4. Let $\varepsilon>0$. There exists a Lipschitz homeomorphism $f: B(0,1) \rightarrow B\left(0, \frac{1}{e}\right)$ of finite distortion such that $K \in L^{1}(B(0,1))$ and $\log \left(e+\frac{1}{J_{f}}\right) \notin L_{\text {loc }}^{1+\varepsilon}(B(0,1))$.

Proof. Without loss of generality we can suppose that $\varepsilon<1$. Set $f(0)=0$ and

$$
f(x)=\frac{x}{\|x\|} \exp \left(\frac{-1}{\|x\|^{2-\varepsilon}}\right) \text { for } x \neq 0 .
$$

From Lemma 2.3 we obtain by elementary computations that

$$
K(x) \sim \frac{1}{\|x\|^{2-\varepsilon}} \text { and } \log \left(e+\frac{1}{J_{f}(x)}\right) \sim \frac{1}{\|x\|^{2-\varepsilon}}
$$


and the result easily follows.

\section{EXAMPLES OF HOMEOMORPHISMS}

We begin with the construction related to Theorem 4.1. In [18] it was observed that homeomorphisms which map suitable regular Cantor sets to each other serve as critical examples for condition N, under integrability conditions on the distortion function. Such a homeomorphism is constructed by changing one Cantor construction to another one by means of mapping "squares to squares". This trick dates back at least to [27],[10]. For our purposes, this approach is not sufficient. Instead of this, we consider product Cantor sets $E_{1} \times E_{2}$ and map one construction to another one by means of mapping "rectangles to rectangles".

Example 7.1. Let $p \in(1, \infty), 1<q<2, \varepsilon>0$ and set $a=\frac{(q-1) p}{p+q-2}$. There is a homeomorphism $f: Q_{0} \rightarrow Q_{0}$ of finite distortion such that $f \in W^{1, p}\left(Q_{0}, \mathbb{R}^{2}\right)$ and $K^{a} \in L^{1}\left(Q_{0}\right)$, but $f^{-1} \notin W_{\text {loc }}^{1, q+\varepsilon}\left(Q_{0}, \mathbb{R}^{2}\right)$.

Proof. We will first give two Cantor constructions in $Q_{0}$. Later $f$ will be defined as the limit of a sequence of piecewise continuously differentiable homeomorphisms $f_{k}: Q_{0} \rightarrow Q_{0}$, where each $f_{k}$ maps the $k$-th step of the first Cantor construction onto the $k$-th step of the second Cantor construction.

We set $a_{0}=b_{0}=c_{0}=d_{0}=\frac{1}{2}$ and

$$
\begin{aligned}
& \alpha=1, \beta=\frac{p+1}{p-1}, \gamma=1+\frac{2}{q-1}, \delta=1+\min \left\{\frac{2 \varepsilon}{q-1}, \frac{1}{p-1}\right\}, \\
& a_{k}=\frac{1}{2^{k+3} k^{\alpha}}, \quad b_{k}=\frac{1}{2^{k+3} k^{\beta}}, \quad c_{k}=\frac{1}{2^{k+3} k^{\gamma}} \text { and } d_{k}=\frac{1}{2^{k+3} k^{\delta}} \text { for } k \in \mathbb{N} .
\end{aligned}
$$

Without loss of generality, we will suppose that $\varepsilon<1$, and thus $\delta<\gamma$. Hence we have the inequalities

$$
\alpha<\beta, \alpha<\gamma, \delta<\beta \text { and } \delta<\gamma .
$$

It is not difficult to verify the following properties :

(i) $a_{k}<\frac{1}{2} a_{k-1}, \quad b_{k}<\frac{1}{2} b_{k-1}, c_{k}<\frac{1}{2} c_{k-1}, d_{k}<\frac{1}{2} d_{k-1}$ for $k \in \mathbb{N}$;

(ii) $a_{k} \sim a_{k-1}, b_{k} \sim b_{k-1}, c_{k} \sim c_{k-1}, d_{k} \sim d_{k-1}$ for $k \in \mathbb{N}$;

(iii) $\lim _{k \rightarrow \infty} 2^{k} a_{k}=\lim _{k \rightarrow \infty} 2^{k} b_{k}=\lim _{k \rightarrow \infty} 2^{k} c_{k}=\lim _{k \rightarrow \infty} 2^{k} d_{k}=0$.

Let

$$
V=\{(1,1),(-1,1),(1,-1),(-1,-1)\} .
$$

The sets $V^{k}=V \times \ldots \times V, k \in \mathbb{N}$, will serve as the sets of indices for our construction. Set $z_{0}=\left[\frac{1}{2}, \frac{1}{2}\right]$. For $v \in V^{1}=V$, let

$$
z_{v}=z_{0}+\frac{1}{4} v, S_{v}=R\left(z_{v}, \frac{1}{4}, \frac{1}{4}\right) \text { and } R_{v}=R\left(z_{v}, a_{1}, b_{1}\right) .
$$



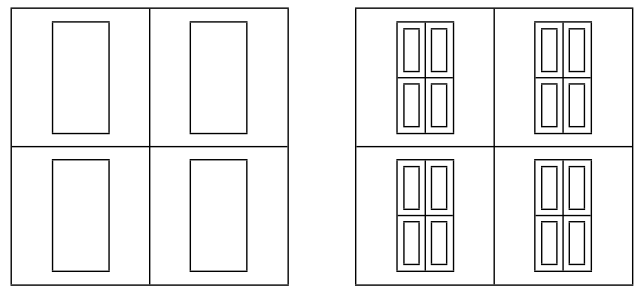

Fig. 1. Rectangles $R_{v}$ for $v \in V^{1}$ and $v \in V^{2}$.

If $k \in \mathbb{N} \backslash\{1\}, w \in V^{k-1}$ and $R_{w}=R\left(z_{w}, a_{k-1}, b_{k-1}\right)$ is a rectangle from the previous step of construction, then $R_{w}$ is divided into four rectangles of equal size and inside them we find concentric rectangles with side lengths $a_{k}$ and $b_{k}$. Thus, for $v=\left(v_{1}, \ldots, v_{k}\right) \in V^{k}$, we set

$$
\begin{aligned}
z_{v} & =z_{w}+\frac{1}{2} a_{k-1}\left(v_{k}\right)_{1} \mathbf{e}_{1}+\frac{1}{2} b_{k-1}\left(v_{k}\right)_{2} \mathbf{e}_{2} \\
& =z_{0}+\frac{\mathbf{e}_{1}}{2} \sum_{j=1}^{k} a_{j-1}\left(v_{j}\right)_{1}+\frac{\mathbf{e}_{2}}{2} \sum_{j=1}^{k} b_{j-1}\left(v_{j}\right)_{2} \\
S_{v} & =R\left(z_{v}, \frac{a_{k-1}}{2}, \frac{b_{k-1}}{2}\right), R_{v}=R\left(z_{v}, a_{k}, b_{k}\right) .
\end{aligned}
$$

See Figure 1. It follows from $(7.3)(i)$ that $R_{v} \subset S_{v}$. The resulting Cantor set

$$
E=\bigcap_{k \in \mathbb{N}} \bigcup_{v \in V^{k}} R_{v}
$$

is clearly a product of two one-dimensional Cantor sets $E=E_{1} \times$ $E_{2}$. From (7.3) $(i i i)$ we see that $E_{1}$ and $E_{2}$ have zero one-dimensional measure; therefore $E$ has zero measure.

The second Cantor construction is similar to the first one; namely for $v \in V^{k}$ we set

$$
\begin{aligned}
& \tilde{z}_{v}=z_{0}+\frac{\mathbf{e}_{1}}{2} \sum_{j=1}^{k} c_{j-1}\left(v_{j}\right)_{1}+\frac{\mathbf{e}_{2}}{2} \sum_{j=1}^{k} d_{j-1}\left(v_{j}\right)_{2} \\
& \tilde{S}_{v}=R\left(\tilde{z}_{v}, \frac{c_{k-1}}{2}, \frac{d_{k-1}}{2}\right), \tilde{R}_{v}=R\left(\tilde{z}_{v}, c_{k}, d_{k}\right) .
\end{aligned}
$$

We are now ready to define the mappings $f_{k}$. Define $f_{0}=\left.\mathrm{id}\right|_{Q_{0}}$. The mapping $f_{1}$ stretches each rectangle $R_{v}, v \in V^{1}$, homogenuously so that $f_{1}\left(R_{v}\right)=\tilde{R}_{v}$. The set $S_{v} \backslash R_{v}$ is stretched to $\tilde{S}_{v} \backslash \tilde{R}_{v}$ so that the resulting map is a homeomorphism.

In the general step (i.e. $k>1$ ), $f_{k}$ is defined as $f_{k-1}$ outside the union of all rectangles $S_{v}, v \in V^{k-1}$. Further, it stretches each rectangle $R_{v}$ to $\tilde{R}_{v}$ homogenuously. We denote by $A_{v}$ two parallelepipeds in $S_{v} \backslash R_{v}$ and the remaining two parallelepipeds are denoted by $B_{v}$ (see Figure 
2.). These parallelepipeds are mapped to the corresponding ones in $\tilde{S}_{v} \backslash \tilde{R}_{v}$ so that the entire map $f_{k}$ is a homeomorphism.
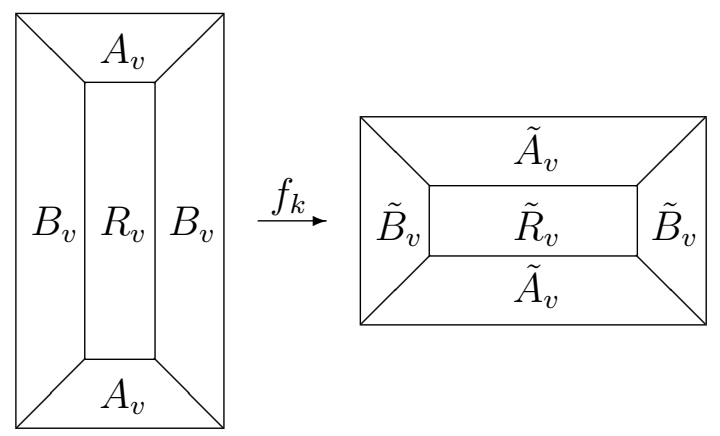

Fig. 2. The mapping $f_{k}$ acting on $S_{v}$

To be more precise, let $f_{0}=\left.\mathrm{id}\right|_{Q_{0}}$, and for $k \in \mathbb{N}$ define (7.4)

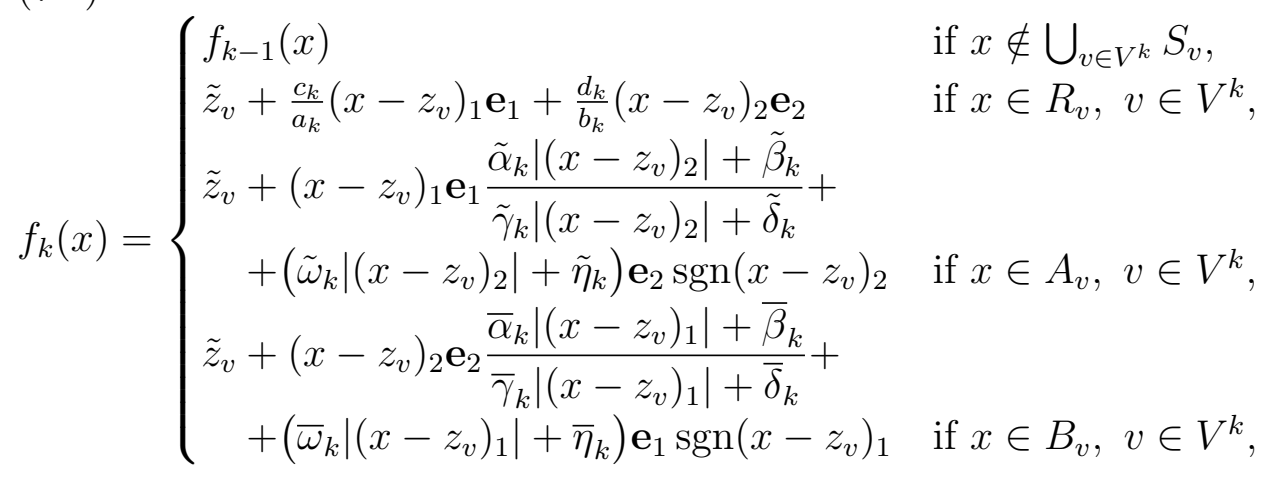

where the constants $\tilde{\alpha}_{k}, \tilde{\beta}_{k}, \tilde{\gamma}_{k}, \tilde{\delta}_{k}, \tilde{\omega}_{k}, \tilde{\eta}_{k}, \bar{\alpha}_{k}, \bar{\beta}_{k}, \bar{\gamma}_{k}, \bar{\delta}_{k}, \bar{\omega}_{k}$ and $\bar{\eta}_{k}$ are chosen so that

$$
\begin{array}{cc}
\tilde{\alpha}_{k} \frac{b_{k-1}}{2}+\tilde{\beta}_{k}=\frac{c_{k-1}}{2}, & \tilde{\alpha}_{k} b_{k}+\tilde{\beta}_{k}=c_{k}, \\
\tilde{\gamma}_{k} \frac{b_{k-1}}{2}+\tilde{\delta}_{k}=\frac{a_{k-1}}{2}, & \tilde{\gamma}_{k} b_{k}+\tilde{\delta}_{k}=a_{k}, \\
\tilde{\omega}_{k} \frac{b_{k-1}}{2}+\tilde{\eta}_{k}=\frac{d_{k-1}}{2}, & \tilde{\omega}_{k} b_{k}+\tilde{\eta}_{k}=d_{k}, \\
\bar{\alpha}_{k} \frac{a_{k-1}}{2}+\bar{\beta}_{k}=\frac{d_{k-1}}{2}, & \bar{\alpha}_{k} a_{k}+\bar{\beta}_{k}=d_{k}, \\
\bar{\gamma}_{k} \frac{a_{k-1}}{2}+\bar{\delta}_{k}=\frac{b_{k-1}}{2}, & \bar{\gamma}_{k} a_{k}+\bar{\delta}_{k}=b_{k}, \\
\bar{\omega}_{k} \frac{a_{k-1}}{2}+\bar{\eta}_{k}=\frac{c_{k-1}}{2}, & \bar{\omega}_{k} a_{k}+\bar{\eta}_{k}=c_{k} .
\end{array}
$$

It is then not difficult to check that $f_{k}$ is a homeomorphism.

Clearly, the limit $f=\lim _{k \rightarrow \infty} f_{k}$ is a homeomorphism of $Q_{0}$ onto $Q_{0}$, differentiable almost everywhere and absolutely continuous on almost 
REGULARITY OF THE INVERSE OF A PLANAR HOMEOMORPHISM 17

all lines parallel to coordinate axes (recall that both $E_{1}$ and $E_{2}$ are of one-dimensional measure zero).

Let $k \in \mathbb{N}$ and $v \in V^{k}$. Since $a_{k} \sim a_{k-1}$ and $b_{k} \sim b_{k-1}$ it is not difficult to find out that the measures of areas $A_{v}$ and $B_{v}$ from Figure 2 satisfy

$$
\left|A_{v}\right| \sim a_{k}\left(\frac{1}{2} b_{k-1}-b_{k}\right) \text { and }\left|B_{v}\right| \sim\left(\frac{1}{2} a_{k-1}-a_{k}\right) b_{k} .
$$

We need to estimate the derivative of our mapping $f_{k}$. We denote its coordinate functions by $f_{k}^{1}$ and $f_{k}^{2}$, i.e. $f_{k}=\left(f_{k}^{1}, f_{k}^{2}\right)$. The function

$$
h(t)=\frac{\tilde{\alpha}_{k} t+\tilde{\beta}_{k}}{\tilde{\gamma}_{k} t+\tilde{\delta}_{k}}=\tilde{\alpha}_{k}\left(\frac{1}{\tilde{\gamma}_{k}}+\frac{\frac{\tilde{\beta}_{k}}{\tilde{\alpha}_{k}}-\frac{\tilde{\delta}_{k}}{\tilde{\gamma}_{k}}}{\tilde{\gamma}_{k} t+\tilde{\delta}_{k}}\right)
$$

is clearly either non-increasing or non-decreasing for $t \in\left[b_{k}, \frac{b_{k-1}}{2}\right]$. Let $x \in \operatorname{int} A_{v}$. Then $\left|\left(x-z_{v}\right)_{2}\right| \in\left[b_{k}, \frac{b_{k-1}}{2}\right]$ and therefore (7.4) (7.5) and (7.3) $(i i)$ give us

$$
\frac{\partial f_{k}^{1}(x)}{\partial x_{1}} \sim h\left(\left|\left(x-z_{v}\right)_{2}\right|\right) \sim \frac{c_{k}}{a_{k}} .
$$

From $t \in\left[b_{k}, \frac{b_{k-1}}{2}\right],(7.5)$ and (7.3) (ii) we also have that $\tilde{\gamma}_{k} t+\tilde{\delta}_{k} \sim a_{k}$. Together with (7.4) and (7.8) this implies

$$
\left|\frac{\partial f_{k}^{1}(x)}{\partial x_{2}}\right|=\left|\left(x-z_{v}\right)_{1}\right|\left|h^{\prime}\left(\left|\left(x-z_{v}\right)_{2}\right|\right)\right| \sim\left|\left(x-z_{v}\right)_{1}\right| \tilde{\alpha}_{k}\left|\frac{\tilde{\beta}_{k}}{\tilde{\alpha}_{k}}-\frac{\tilde{\delta}_{k}}{\tilde{\gamma}_{k}}\right| \frac{\tilde{\gamma}_{k}}{a_{k}^{2}} .
$$

Hence

$$
D f(x)=D f_{k}(x) \sim\left(\begin{array}{cc}
\frac{c_{k}}{a_{k}} & \pm\left(x-z_{v}\right)_{1}\left(\tilde{\beta}_{k} \tilde{\gamma}_{k}-\tilde{\delta}_{k} \tilde{\alpha}_{k}\right) \frac{1}{a_{k}^{2}} \\
0 & \tilde{\omega}_{k}
\end{array}\right)
$$

for every $x \in \operatorname{int} A_{v}$. Analogously we can check that

$$
D f(y) \sim\left(\begin{array}{cc}
\bar{\omega}_{k} & 0 \\
\pm\left(y-z_{v}\right)_{2}\left(\bar{\beta}_{k} \bar{\gamma}_{k}-\bar{\delta}_{k} \bar{\alpha}_{k}\right) \frac{1}{b_{k}^{2}} & \frac{d_{k}}{b_{k}}
\end{array}\right)
$$

for every $y \in \operatorname{int} B_{v}$.

Since $k^{\xi}-(k+1)^{\xi} \sim k^{\xi-1}$ for every $\xi \neq 0$ we obtain with the help of (7.5) and (7.1) that $\tilde{\omega}_{k} \sim \frac{\frac{d_{k-1}}{2}-d_{k}}{\frac{b_{k-1}}{2}-b_{k}} \sim k^{\beta-\delta}$ and

$$
\left|\tilde{\beta}_{k} \tilde{\gamma}_{k}-\tilde{\delta}_{k} \tilde{\alpha}_{k}\right|=\left|c_{k} \frac{\frac{a_{k-1}}{2}-a_{k}}{\frac{b_{k-1}}{2}-b_{k}}-a_{k} \frac{\frac{c_{k-1}}{2}-c_{k}}{\frac{b_{k-1}}{2}-b_{k}}\right| \leq C \frac{k^{\beta-\alpha-\gamma}}{2^{k}} .
$$

For $x \in \operatorname{int} A_{v}$ we clearly have $\left|\left(x-z_{v}\right)_{1}\right| \leq a_{k-1}$ and therefore (7.9), (7.1) and (7.2) give us

$$
|D f(x)| \sim \max \left\{k^{\alpha-\gamma}, k^{\beta-\gamma}, 0, k^{\beta-\delta}\right\}=k^{\beta-\delta} \text { and } K(x) \sim \frac{k^{\beta-\delta}}{k^{\alpha-\gamma}}
$$


and analogously for $y \in \operatorname{int} B_{v}$ we conclude from (7.10) and (7.2) that

$$
|D f(y)| \sim \max \left\{k^{\alpha-\gamma}, 0, k^{\alpha-\delta}, k^{\beta-\delta}\right\}=k^{\beta-\delta} \text { and } K(y) \sim \frac{k^{\beta-\delta}}{k^{\alpha-\gamma}}
$$

Therefore (7.7), (7.11), (7.12) and (7.1) give us

$$
\begin{aligned}
\int_{Q_{0}}|D f|^{p} & \leq C \sum_{k \in \mathbb{N}} \sum_{v \in V^{k}}\left(\left|A_{v}\right|+\left|B_{v}\right|\right) k^{(\beta-\delta) p} \\
& \leq C \sum_{k \in \mathbb{N}} 2^{2 k} \frac{1}{2^{2 k} k^{1+\beta+\alpha}} k^{(\beta-\delta) p} \\
& \leq C \sum_{k \in \mathbb{N}} \frac{1}{k^{1+p \min \left\{\frac{2 \varepsilon}{q-1}, \frac{1}{p-1}\right\}}}<\infty
\end{aligned}
$$

and

$$
\begin{aligned}
\int_{Q_{0}} K^{a} & \leq C \sum_{k \in \mathbb{N}} \sum_{v \in V^{k}}\left(\left|A_{v}\right|+\left|B_{v}\right|\right) k^{(\beta+\gamma-\delta-\alpha) a} \\
& \leq C \sum_{k \in \mathbb{N}} 2^{2 k} \frac{1}{2^{2 k} k^{1+\beta+\alpha}} k^{(\beta+\gamma-\delta-\alpha) \frac{(q-1) p}{p+q-2}} \\
& \leq C \sum_{k \in \mathbb{N}} \frac{1}{k^{1+\frac{(q-1) p}{p+q-2} \min \left\{\frac{2 \varepsilon}{q-1}, \frac{1}{p-1}\right\}}<\infty .}
\end{aligned}
$$

Let $k \in \mathbb{N}, v \in V^{k}$ and $\tilde{x} \in \operatorname{int} \tilde{A}_{v}$, i.e. there is $x \in \operatorname{int} A_{v}$ such that $\tilde{x}=f(x)$. Clearly $J_{f^{-1}}(\tilde{x})>0$ and thus $D\left(f^{-1}\right)(\tilde{x})=(D f(x))^{-1}$. From this, (7.9) and

$$
\left(\begin{array}{cc}
A & B \\
0 & C
\end{array}\right)^{-1}=\left(\begin{array}{cc}
\frac{1}{A} & \frac{-B}{A C} \\
0 & \frac{1}{C}
\end{array}\right) \text { for any } A, B, C \neq 0
$$

we obtain with the help of (7.11) and (7.2) that

$$
\left|D\left(f^{-1}\right)(\tilde{x})\right| \sim \max \left\{k^{\gamma-\alpha}, k^{\beta-\gamma-(\alpha-\gamma)-(\beta-\delta)}, 0, k^{\delta-\beta}\right\}=k^{\gamma-\alpha} .
$$

Similarly we can check that $\left|D\left(f^{-1}\right)(\tilde{y})\right| \sim k^{\gamma-\alpha}$ for every $\tilde{y} \in \operatorname{int} \tilde{B}_{v}$. Thus (7.1) gives us

$$
\begin{aligned}
\int_{Q_{0}}\left|D\left(f^{-1}\right)\right|^{q+\varepsilon} & \geq C \sum_{k \in \mathbb{N}} \sum_{v \in V^{k}}\left(\left|\tilde{A}_{v}\right|+\left|\tilde{B}_{v}\right|\right) k^{(\gamma-\alpha)(q+\varepsilon)} \\
& \geq C \sum_{k \in \mathbb{N}} 2^{2 k} \frac{1}{2^{2 k} k^{1+\gamma+\delta}} k^{(\gamma-\alpha)(q+\varepsilon)} \\
& \geq C \sum_{k \in \mathbb{N}} \frac{1}{k^{1+1+\frac{2}{q-1}+1+\frac{2 \varepsilon}{q-1}}} k^{\frac{2}{q-1}(q+\varepsilon)}=C \sum_{k \in \mathbb{N}} \frac{1}{k}=\infty
\end{aligned}
$$

Proof of Example 1.4. Set $q=1+\frac{\delta}{2}$ and $\varepsilon=\frac{\delta}{2}$. We can clearly find $p>1$ small enough such that $(q-1) \frac{p}{p+q-2}>1-\delta$; therefore the statement follows from Example 7.1. 
The assumptions of Example 7.1 left open the case when $f$ is Lipschitz. This is actually easier: consider

$$
f(x, y)=\left(x,|y|^{\alpha} \operatorname{sgn} y\right) .
$$

Given $1<q<2$ and $\varepsilon>0$, we find $\alpha>1$ such that

$$
(\alpha-1)(q-1)<1 \text { and } \frac{1-\alpha}{\alpha}(q+\varepsilon)<-1 .
$$

With this choice, $K \in L^{q-1}\left(Q_{0}\right)$, but $f^{-1} \notin W_{\text {loc }}^{1, q+\varepsilon}\left(Q_{0}, \mathbb{R}^{2}\right)$.

\section{REFERENCES}

[1] K. Astala, T. Iwaniec, G. Martin and J. Onninen, Extremal mappings of finite distortion, preprint.

[2] J. Ball, Global invertibility of Sobolev functions and the interpenetration of matter, Proc. Roy. Soc. Edinburgh Sect. A 88 no. 3-4 (1981), 315-328.

[3] M. A. Brakalova and J. A. Jenkins, On solutions of the Beltrami equation, $J$. Anal. Math. 76 (1998), 67-92.

[4] G. David, Solutions de l'equation de Beltrami avec $\|\mu\|_{\infty}=1$, Ann. Acad. Sci. Fenn. Ser. A I, Math. 13 no. 1 (1988), 25-70.

[5] C. Dellacherie and P. A. Meyer, Probabilities and potential, North-Holland Mathematics Studies, 29, North-Holland Publishing Co., 1978.

[6] D. Faraco, P. Koskela and X. Zhong, Mappings of finite distortion: The degree of regularity, to appear in Adv. Math.

[7] H. Federer, Geometric measure theory, Die Grundlehren der mathematischen Wissenschaften, Band 153 Springer-Verlag, New York, 1969 (Second edition 1996).

[8] I. Fonseca and W. Gangbo, Degree Theory in Analysis and Applications, Clarendon Press, Oxford, 1995.

[9] F. W. Gehring and O. Lehto, On the total differentiability of functions of a complex variable, Ann. Acad. Sci. Fenn. Ser. A I 272 (1959), 1-9.

[10] F. W. Gehring and J. Väisälä, Hausdorff dimension and quasiconformal mappings, J. London Math. Soc. 6 (1973), 504-512.

[11] V. Gutlyanskii, O. Martio, T. Sugawa and M. Vuorinen, On the Degenerate Beltrami Equation, to appear in Trans. Amer. Math. Soc.

[12] J. Heinonen and P. Koskela, Sobolev mappings with integrable dilatations, Arch. Rational Mech. Anal. 125 no. 1 (1993), 81-97.

[13] S. Hencl, P. Koskela and J Onninen, A note on extremal mappings of finite distortion, preprint.

[14] T. Iwaniec and G. Martin, Geometric function theory and nonlinear analysis, Oxford Mathematical Monographs, Clarendon Press, Oxford 2001.

[15] T. Iwaniec and G. Martin, Beltrami equation, to appear in Mem. Amer. Math. Soc.

[16] T. Iwaniec and V. Šverák, On mappings with integrable dilatation, Proc. Amer. Math. Soc. 118 (1993), 181-188.

[17] J. Kauhanen, An example concerning the zero set of the Jacobian, preprint.

[18] J. Kauhanen, P. Koskela and J. Malý, Mappings of finite distortion: Condition N, Michigan Math. J. 49 (2001), 169-181.

[19] J. Kauhanen, P. Koskela and J. Malý, Mappings of finite distortion: Discreteness and openness, Arch. Ration. Mech. Anal. 160 (2001), 135-151. 
[20] J. Kauhanen, P. Koskela, J. Malý, J. Onninen and X. Zhong, Mappings of finite distortion: Sharp Orlicz-conditions, Rev. Mat. Iberoamericana 19 (2003), 857872 .

[21] P. Koskela and J. Malý, Mappings of finite distortion: the zero set of the Jacobian, J. Eur. Math. Soc. 5 no. 2 (2003), 95-105.

[22] P. Koskela and J. Onninen, Mappings of finite distortion: Capacity and modulus inequalities, preprint.

[23] J. Malý, Lectures on change of variables in integral, Preprint 305, Department of Math., University of Helsinki, 2001.

[24] G. Moscariello, On the integrability of the Jacobian in Orlicz spaces, Math. Japanica 40 (1992), 323-329.

[25] S. Müller, Higher integrability of determinants and weak convergence in $L^{1}$, J. Reine Angew. Math. 412 (1990), 20-34.

[26] S. Müller, Q. Tang and B. S. Yan, On a new class of elastic deformations not allowing for cavitation, Ann. Inst. H. Poincaré Anal. Non Lináire 11 (1994), 217-243.

[27] S. Ponomarev, Examples of homeomorphisms in the class $A C T L^{p}$ which do not satisfy the absolute continuity condition of Banach, Dokl. Akad. Nauk USSR 201 (1971), 1053-1054.

[28] S. Rickman, Quasiregular mappings, Ergebnisse der Mathematik und ihrer Grenzgebiete (3) [Results in Mathematics and Related Areas (3)], 26. SpringerVerlag, Berlin, 1993.

[29] V. Ryazanov, U. Srebro, and E. Yakubov, BMO-Quasiconformal mappings, J. Analyse Math. 83 (2001), 1-20.

[30] Q. Tang, Almost-everywhere injectivity in nonlinear elasticity, Proc. Roy. Soc. Edinburgh Sect. A 109 no. 1-2 (1988), 79-95.

Department of Mathematics and Statistics, University of Jyväskylä, P.O. Box 35 (MAD), FIN-40014, JyväSKYlä, Finland

E-mail address: hencl@maths.jyu.fi

E-mail address: pkoskela@maths.jyu.fi 